\title{
Rock Deformation Laboratory Experiments
}

\section{THOMAS KING ${ }^{1}{ }^{*}$, PHILIP BENSON ${ }^{2}$, LUCA DE SIENA ${ }^{3}$, AND SERGIO VINCIGUERRA ${ }^{1}$}

$4 \quad{ }^{1}$ Department of Earth Sciences, University of Turin, Via Tommaso Valperga Caluso, 35, 10125

5 Turin, Italy; sergiocarmelo.vinciguerra@unito.it.

$6 \quad{ }^{2}$ Rock Mechanics Laboratory, School of Earth and Environmental Sciences, University of

7 Portsmouth, Burnaby Building, Portsmouth PO1 3QL, UK; philip.benson@port.ac.uk

$8 \quad{ }^{3}$ Institute of Geosciences, Johannes Gutenberg University, Mainz, Germany; 1desiena@uni9 mainz.de.

10 Corresponding author: Thomas King (thomas.king@edu.unito.it)

11 Abstract

We report a new method using a Time Delay Neural Network to transform Acoustic

13 Emission (AE) waveforms into timeseries of instantaneous frequency content and permutation entropy. This permits periods of noise to be distinguished from signals. The model is trained in sequential batches, using an automated process that steadily improves signal recognition as new data are added. The model was validated using AE data from rock deformation experiments, using Darley Dale Sandstone in fully drained conditions at a confining pressure of $20 \mathrm{MPa}$ (approximately $800 \mathrm{~m}$ simulated depth). The model is initially trained by manual picking of five high amplitude waveforms randomly selected from the dataset (experiment). This is followed by semi-supervised training on a subset of 300 waveforms. When compared with the standard amplitude-threshold picking methodology the source locations obtained from the time difference of arrivals show $\sim 400 \%$ increase in the number of events located, $\sim 150 \%$ increase in signal to noise ratios and a $\sim 30 \%$ reduction in location residuals. 
Acoustic emissions (AE) are the transient elastic waves produced by the sudden redistribution (release) of stress when a material fractures. These signals are considered to represent the laboratory analogue of natural earthquakes with wider applications spanning from non-destructive testing to vibration monitoring. In deformation experiments, $\mathrm{AE}$ are related to the initiation and growth of fractures (e.g. Lockner et al., 1992), matrix cracking (Scholey et al., 2010), fluid flow through fractures (Benson et al., 2019; Fazio et al., 2019), and fibre breakage and various debonding processes (Bohse, 2000). Detection of these signals is a valuable asset as they provide a non-destructive and immediate feedback to dynamically evolving systems without the need for interference. However, a limitation of AE analysis is that results are often dependent on the successful discrimination of signals from the background noise. As datasets are often very large, therefore any approach requires an automated approach that is robust enough to handle to waveforms in a medium that is continually evolving.

The simplest processing method to extract basic data uses stepwise calculation along the waveform timeseries of energy ratios ("signal-to-noise" ratio). This type of analysis is often used to extract discrete time series from a much longer (quasi-continuous) signal, with triggering dependent on the arrival of large amplitude spikes (such as the first arrival of an earthquake). Whilst intuitive and less computationally demanding than other techniques, this method is prone to errors, as the largest signal-to-noise ratio may not refer to the first arrival of a genuine signal (Guoping et al., 2004; Pomponi \& Vinogradov, 2013). To reduce these false triggers, methods that take advantage of the inherent characteristics of microseismic signals, 46 i.e., frequency content, have been proposed (Zhang et al., 2013). These methods use an 47 automated approach via the Hilbert-Huang Transform (HHT, Huang et al., 1998) to identify low-frequency AE signals from high-frequency background noise. A further refinement of this 
the first arrival of seismic waves (Jia et al., 2015).

Entropy-based techniques, which identify the incoherent nature of the noise signal by relating data to past and previous values, are also well documented. In medicine, Ródenas et al. (2015) reported the accurate detection of atrial fibrillation from electrocardiograms. A similar approach uses intrinsic mode functions (IMF) to recognise focal electroencephalogram signals (Sharma et al., 2015). Similar techniques have found their use at the field scale. For example, fluctuations in entropy were detected prior to the Mexican earthquake of 2017 (Ramírez-Rojas et al., 2018). Entropy is highly sensitive to seismic amplitude, frequency and phase changes (Jia et al., 2019). They demonstrate immunity to noise under a wide range of environmental conditions due to a sensitivity to the coherency of a real signal, making them uniquely suited for seismological analyses. The technique can also be applied to active seismic data (Zoukaneri \& Porsani, 2015) by measuring the entropy of the instantaneous frequency content, increasing the level of information available for sub-surface mapping. defines when a time-period is "signal" or "noise". Often, this threshold is set based on the user experience and knowledge of the recording equipment. When tools are not calibrated, as is often the case in AE data (e.g. Høgsberg \& Krenk, 2015), such an approach remains imprecise and will lead to further systematic errors regardless of the technique. Nevertheless, advances in machine learning and artificial intelligence tools are now ushering a new era of data analysis, which seeks to minimise biases enforced by user-defined parameters. Time Delay Neural Networks (TDNN) are an automated signal classification technique that are designed with the purpose of identifying patterns and trends in shift-invariant time-series data without explicitly knowing the beginning or end of a signal (Derakhshani \& Schuckers, 2004; Peddinti et al., 2015; Waibel et al., 1995). Analogous to a 1D Convolutional Neural Network (CNN), TDNNs are a form of Recurrent Neural Network (RNN) that models the propagation characteristics of 
time series data similar to entropy techniques. By constructing models of the key elements of audio, or elastic vibrations in the case of seismology, they can recognise different speakers when employed for speech recognition (Haffner \& Waibel, 1992) and perform robustly in the presence of reverberations (Snyder et al., 2015).

Here, we seek to take advantage of TDNNs by applying a simple noise or signal classification routine to $\mathrm{AE}$ data from a typical rock-deformation experiment using Darley Dale Sandstone, where the TDNN is used to define the onset of energy as the boundary between the two classifications. By iteratively training a neural network on calculated timeseries of seismic envelopes, instantaneous frequency content and estimated signal entropy, the method picks AE waveforms and automatically incorporates them into the evolving (updated) model, thus improving later picks. To validate our approach, AE source locations are solved using the pick times obtained from the trained model using a Time Difference of Arrival method (e.g. Comanducci et al., 2020). AE distribution and error parameters are then compared to those obtained from picking signals with a simple amplitude threshold method.

\section{Data and Method}

\section{Acoustic Emission Data}

A 40x100 mm cylindrical sample of Darley Dale Sandstone was deformed using a conventional triaxial deformation apparatus (Fazio, 2017) at a confining pressure of $20 \mathrm{MPa}$, representing approximately $800 \mathrm{~m}$ depth. To ensure the experiment collected an AE dataset that contains a large range of amplitudes (Nakamura et al., 1972) and fracture mechanisms (Stanchits et al., 2006), the sample was deformed at a deformation rate of $3.6 \mathrm{~mm} / \mathrm{hr}$ until brittle failure following the protocol of Fazio et al., (2017). For a detailed overview of the AE data acquisition methodology, please refer to Appendix 1.

\section{Model Parameters}


A previous study had proposed using only the waveforms as input data (Derakhshani \&

100 Schuckers, 2004). After extensive testing, that approach was found to perform less robustly

101 with the high noise conditions of the AE measured here. Instead, we focus on the simple

102 attributes of signal amplitude and frequency content as training parameters to classify the

103 waveform. The former is modelled through the seismic envelope, whilst the latter by

104 instantaneous frequency content obtained through the HHT. A third parameter, permutation entropy (Unakafova \& Keller, 2013), encompasses both aspects. A fourth timeseries, defined as the target model output, is used to classify the AE waveforms through binary separation of noise and signal data, assigned -1 and 1 respectively. Signal is defined as the time period between the calculated onset of energy and the point at which energy drops below a pre-pick noise threshold. Due to uncertainties in the waveform content following the main arrival (i.e. reflections) the model is only trained on noise identified during the pre-signal period.

112 the instantaneous energy of the signal (Figure 1a). Typically, noise is represented by low 113 amplitude values and signal by high amplitude values. The boundary between these two is often 114 defined by a rapid increase in values as a signal arrives. However, in the presence of low amplitude data, or strong scattering where the envelope of the signal can become broadened, the increase becomes shallower or simply not present (De Siena et al., 2016). To address this,

117 the HHT provides a measure of instantaneous frequency content along the entire time series

118 (Figure 1b) that is independent of amplitude, therefore any boundary between classifications 119 is still preserved when energies are low. Noise is identified through a dominance of high120 frequency energy, whilst the signal is identified through a consistent dominance of low 121 frequency waves. However, on its own, this method is likely to fail in the presence of highfrequency signals that are similar in content to the background. 
124 degree of irregularity of a random timeseries (Figure 1c). Ordinal patterns are calculated that represent the relationship between past and future values at each time step of the waveform

126 (i.e. an increasing trend). A probability density function of the number of patterns within a

127 moving window is then calculated, thus removing any dependence on the amplitude of the 128 original waveform (Unakafova \& Keller, 2013). The larger the value of permutation entropy,

129 the higher the diversity of ordinal patterns is and the more complex the input data are. Thus, 130 noise is characterised by high values and signal by low, however, the boundary is slightly

131 shifted to that of the seismic envelope. A more detailed overview on the training data is 132 provided in Appendix 2.

\section{Training Routine}

134 Similar to the previously described entropy method, a TDNN models the temporal trend 135 (or trajectory) of the training data, classifying patterns in the data according to the target timeseries. A key difference of a TDNN from other multi-layer neural networks is that patterns are classified with shift-invariance; a specific pattern may occur at any point in the timeseries. With each successive layer in the network, increasingly coarse trends in the training data are identified, therefore ensuring the model learns the most important features of the chosen 140 classifications.

Five high-amplitude waveforms are randomly selected from an AE pool to create an initial model that is trained under supervised conditions. Their onsets are manually picked, and the model iteratively trained on the concatenated input parameters (training dataset) after each

144 waveform. At this stage, the model already has a relatively high degree of picking accuracy.

145 Following this, the model undergoes semi-supervised training. A waveform of any amplitude

146 is randomly selected from the available pool. The input parameters are both calculated and 147 simulated in the neural network model generating an output. 
149 pick the signal onset from the model output due to the uncertain transition from noise to signal.

150 With knowledge of the sample velocities, an effective approach was to define a window 151 between the origin time (or first arrival time when picking subsequent waveforms in a single 152 AE group) and the maximum possible arrival time for energy traversing the length of the 153 sample. Within this window we calculate the instantaneous frequency content of the model output in order to characterise the rapid change in model values as a signal is detected. The onset of energy is then set when the frequencies and model output exceed $14 \mathrm{KHz}$ and -0.95 , respectively.

Once the onset is identified, two 'picking quality' ratios are calculated around this value. A short-term SNR of the original waveform and a SNR of the model output. After extensive testing, we set their values to 2.0 for the former and between 0.3 and 0.9 for the latter as they identified a reliable onset when the training dataset was still small. When both ratios are exceeded, the input parameters are added to the training data and the neural network is updated. To improve computation speed, the model is trained in batches of 10 waveforms. Waveforms can be further down-sampled to increase computation speed, although this will decrease data quality. As this is an iterative process (Figure 2), the model is steadily improved over time, which allows for the later inclusion of data that may not have been initially included. The model ceases training once the training dataset exceeds 300 waveforms as the quality of picking did not improve significantly after this stage.

To provide a robust comparison and best highlight the improvements offered by the proposed neural network approach, AE data are also processed using a simple amplitudethreshold method to pick data. The root mean square (RMS) envelope of each waveform is calculated and the pick time is chosen when the envelope exceeds 1.1 times the level of the background noise (using the same windowing approach as before). 


\section{Source Location}

Pick times are inverted for source location using a Time Difference of Arrival (TDOA) method (Tobias, 1976). Although this method is only robust in the case of weak velocity anisotropy, the TDOA method is well established in micro-seismic source location (e.g. Comanducci et al., 2020) and works well for the near-isotropic Darley Dale sandstone as used here. These data are calculated by pairwise subtraction of the time of arrival values to each sensor from a single source. A fixed velocity is used in the calculation that is updated as time progresses (see Appendix 1). The calculated TDOA values are determined through iterative estimation of the source location. The process iterates the location using the L2 norm of the location residual, comparing between calculated and TDOA values. It arrives to a local minimum at the best determined source location. To be valid, sources are located with a minimum of 6 arrival times, although the accuracy greatly improves with the addition of more sensors.

\section{Results and Discussion}

At high amplitudes, both the neural network (NN) and the amplitude threshold method (AT) performed equivalently. This is unsurprising as the seismic envelope is a key indicator of an AE arrival in both methods. However, as amplitudes decrease and reach the level of the background noise, discrepancies in pick times become increasingly apparent (Figure 3). Whilst it still performed relatively well with impulsive arrivals, the AT method (Figure 3, blue line) struggled with the strongly scattered or emergent arrivals that are characteristic of low amplitude AE data. Such data are challenging for picking methods, as the first arrival of the Pwave becomes more discriminatory due to the convolution of many wave-modes that extend into the coda (e.g. Grosse \& Ohtsu, 2008). The NN on the other hand, was much more consistent in capturing the first arrival regardless of amplitude, wave-mode or waveform 
197 character. By modelling characteristic sequences of data, the TDNN is able to classify the 198 patterns that uniquely define signal or noise in the timeseries. From the model output (Figure

199 3, red line), the arrival time may then be obtained from a far simpler timeseries, even for data 200 that was not picked by the AT method. It should be noted that the windowing scheme was 201 crucial to avoid picking late high energy coda arrivals or even the coda from a prior AE in the pre-pick noise.

Although relevant in all forms of waveform analysis (e.g. seismic, microseismic), the occurrence of external (outside the sample) or electronic noise (often high amplitude, high frequency) with temporal and frequency characteristics similar to real events is a characteristic element of AE data processing. Waveform frequency data has already been shown to be a reliable parameter to characterise waveform data ( $96 \%$ accuracy), but still leads to false-alarms in the pre-pick noise without additional constraints on arrival time (Jiang \& Zheng, 2020). Similar results are obtained when only amplitude data is used (Chen et al., 2019), but such an approach is likely to fail in high-noise environments. The method presented here is distinct from the previously mentioned studies in that it combines multiple input parameters to train the model (amplitude, frequency and entropy). This provided a notable advantage over single feature methods as a more robust characterisation of noise (rather than only signal) was obtained. However, the TDNN performed poorly in the presence of very high amplitude data (SNR $>800$ ). Due to the way the signal is decomposed during the HHT, the noise window is characterised by low frequency information thus resulting in errors during training. As these data occurred very rarely, it was not considered detrimental to the overall model. of the fault plane through the entire experiment (Figure 4). Although events with high location residuals are displayed here for completeness, the distribution of AE hypocentres is consistent 
et al., 1992). Considering the evolution through time (Figure 4b), we note that the event locations are diffuse throughout the sample during the early stages of deformation (windows 1, 2 and 3). As strain increases, during the period of strain-softening (window 4), the distribution of AE locations starts shaping a fault structure (strain localisation). During crack coalescence and brittle failure (window 5) event locations are primarily located along the observed failure plane. Whilst the general distribution of events remain the same for both methods, the inconsistencies in waveform picking that were shown in Figure 3 have resulted in significant differences in source location that can differ by several centimetres for individual AE (Figure 4, coloured dots). A key component of the presented method is that the model is trained on data that came from same the dataset that is due to be picked. As AE datasets are typically very large, it is impractical to manually pick a dataset to truly validate our source locations. Nonetheless, a focusing of events towards a failure plane is much more evident when using picks from the NN compared to the AT method.

In terms of overall performance TDNNs (or RNNs) are generally considered to be equivalent to the more powerful Convolutional Neural Network (CNN) for use in signal detection (G et al., 2018; Guo et al., 2020; Yao et al., 2020). Moreover, the number of training data required to create a well performing model also remains relatively low for both approaches (e.g. Chen et al., 2019). An important difference, however, is that the TDNN presented here outputs a transitional classification rather than the absolute output of a CNN. This provides a measure of uncertainty that is time dependent and thus physically much more appropriate for data generated by time-dependent processes, such as waveform data and for the vast majority of pre-failure processes.

The semi-supervised approach presented here may be considered advantageous due to the low workload requirements when training the model on new datasets. To initiate a new training routine, only 5 high amplitude waveforms needed to be manually picked before the 
model could train itself. In addition, as the model improves more low amplitude data is incorporated into the model automatically, thus removing the requirement to manually pick difficult data. The choice of network, $\mathrm{CNN}$ or TDNN, is therefore tuned to the end-user requirements rather than on any standard performance metrics.

In any case, the models derived using the method proposed here may be applied to new datasets or modified as a triggering tool to use with continuous or real-time data. An important advantage of the TDNN over CNNs is that the input timeseries may be of any length, regardless of those used in the training routine. Rather than identifying individual elements (such as the first arrival), TDNNs characterise sequences of features in order to classify patterns. Thus, a continuous dataset may first be down-sampled to generate a quick approximation of arrival times (trigger) and the exact same model may then be used on the higher resolution recording to obtain a more accurate pick.

\section{Conclusions}

This study has presented a workflow and application of a machine learned waveform picking tool. A time delay neural network is trained to recognise the onset of AE energy using instantaneous frequency, seismic envelope and entropy measurements. Statistical results demonstrate the reliability of the method and highlight the potential of using multiple waveform characterisation techniques to determine the arrival of acoustic energy.

We conclude that an automated process may be readily applied to AE datasets, as recorded here from a triaxial rock deformation experiment. Aside from the 5 waveforms used to initially train the model, no user intervention is required. A high degree of picking accuracy and the inclusion of low-amplitude data that may be missed by traditional single-parameter threshold methods results in datasets with a high source location recovery rate and a reduction in the propagation of errors in further analysis of the data. 
Acoustic Emission data are obtained at the Rock Mechanics Laboratory, University of

273 Portsmouth. Raw data files, pick times, source locations and errors are available at https://zenodo.org/record/3958910. Programming codes were developed in MATLAB® version 2018a and are available at https://github.com/thomaskingunito/programming.

\section{Acknowledgements}

The authors thank Emily Butcher for sample preparation and Peter Ibemesi for assistance during the laboratory experiments. The authors would also like to thank Klaus Regenauer-Lieb and David Dempsey for their helpful comments during the development of the code.

\section{Appendix 1: Rock Deformation Experiment}

Darley dale sandstone (DDS) is ubiquitous in rock mechanics testing. It is a brownyellow, feldspathic sandstone with a modal composition of quartz (69\%), feldspars (26\%), clay

283 (3\%) and mica (2\%) (Heap et al., 2009). Previous studies report a connected porosity of 13.3\% $284 \pm 0.8 \%$ with grain sizes varying from $100-800 \mu \mathrm{m}$ (Zhu \& Wong, 1997). Pore sizes measured by mercury porosimeter have a mean diameter of $10 \mathrm{gm}$, but with significant microporosity (Read et al., 1995). The unconfined compressive strength is $160 \mathrm{MPa}$ (Baud \& Meredith, 1997). At the scale analysed here, no distinct layering or laminations were present. A cylindrical rock sample was cored using a diamond tipped hollow coring drill to prepare a $4 \mathrm{~cm}$ diameter sample that was then trimmed to $10 \mathrm{~cm}$ length with a diamond saw. End faces are accurately ground using a lathe fitted with a cross-cutting diamond grinding disk with surfaces flat and parallel to within $0.01 \mathrm{~mm}$.

Deformation was performed using a conventional triaxial deformation cell installed at

293 the Rock Mechanics Laboratory, University of Portsmouth (Fazio, 2017). The sample 
presented here was deformed until brittle failure at a confining pressure of $20 \mathrm{MPa}$ at a constant deformation rate of $3.6 \mathrm{~mm} / \mathrm{hr}$. Experimentation was performed under fully drained conditions to avoid any fluid-driven effects on AE frequency content (Benson et al., 2010). These environmental conditions ensure that a high number of $\mathrm{AE}$ are obtained and any timedependent variations in the signal waveform are predominantly due to the scattering effects of microfractures, thus allowing for the sampling of a diverse range of deformation structure. Axial displacement is measured with a non-contact Eddy Displacement system mounted to the apparatus. It comprises of three sensors that accurately (sub-micron) measure the distance to a target steel plate attached to the driving piston. These readings are averaged and are used to set the target deformation rate via feedback to an axial stress intensifier.

For AE data acquisition the protocol of Benson et al. (2007) was followed. The dry sample was positioned inside an engineered rubber jacket fitted with ports for an array of twelve $1 \mathrm{MHz}$ single-component Piezo-Electric Transducers (PZTs, model PAC Nano30) were embedded (Figure A1). These sensors have a relatively flat frequency response between 125$750 \mathrm{KHz}$. Sensor output is connected to preamplifiers set to $40 \mathrm{~dB}$, focusing on data quality over quantity. An ITASCA-Image "Milne" recorder operate in a standard 'trigger' model,

310 downloading all twelve channels when any single channel passes a set $100 \mathrm{mV}$ threshold (e.g.

311 Gehne, 2018). During experimentation, in addition to 'passive' mode (recording of fracturing

312 events) the sensors are also used in 'active' mode for P-wave elastic velocity measurements in 313 order to derive a velocity model for source locations (Figure A2). Periodically, each PZT was 314 triggered in sequence with a high voltage $(200 \mathrm{~V})$ pulse, with the energy recorded by the 315 remaining 11 sensors in the array. Signal to noise (SNR) was further improved by pulsing each sensor multiple times (16) and stacking the received waveforms, with each survey of 12sensors generating 144 non-unique raypaths and taking approximately 30 seconds to complete. 
319 of 24360 AE were detected by the 'trigger' recording system (Figure A2, histogram $0.01 \%$

320 strain bins), however, it is unknow what percentage of these signals are noise related.

321 Differential stress increases gradually (Figure A2, black line), entering the elastic phase of 322 deformation at approximately $0.3 \%$ strain, coinciding with a rapid increase in the number of 323 AE recorded. During the period of strain-softening from $0.7-0.9 \%$ strain the rate of $\mathrm{AE}$ 324 dramatically accelerates and peaking at $\sim 1000$ events $/ 0.01 \%$ strain. As the sample goes through 325 peak stress (145 MPa) several pulses in AE count correspond with minor drops in differential stress. However, the overall rate decreases with time. At $\sim 1.4 \%$ strain the sample fails, with Figure 4a highlighting the formation of a fracture plane marked in red.

\section{Appendix 2: Model Training Parameters}

\section{Seismic Envelope}

The Root Mean Square (RMS) envelope provides a scaled amplitude estimate of the AE trace. The envelope represents the instantaneous energy of the signal and is computed in a sliding window of 10 samples. Such a narrow window is selected to avoid smoothing of the low amplitudes at signal onset. In low-noise environments the amplitude of the signal greatly exceeds that of the pre-signal noise and provides a good marker of the onset of energy. However, as noise increases, this onset becomes increasingly difficult to identify resulting in a high degree of uncertainty.

\section{Instantaneous Frequency Content}

Intrinsic modal functions represent simple oscillatory modes of complex signals. Unlike harmonic signals, IMF can have variable frequency and amplitude content through time. Known as empirical mode decomposition (EMD), IMF of individual AE are obtained through a continuous screening process called "sifting" and must satisfy the following requirements: The number of extrema and the number of zero-crossings must either be equal or differ by a 
343 minimum of one, whilst the mean of the upper and lower envelopes is zero. The procedure

344 follows as thus (Huang et al., 1998):

345 1. The local extrema in the time series $X(t)$ are identified

346 2. Local maxima are connected by a cubic spline line to produce an upper envelope

3473 . This is repeated for the local minima to produce a lower envelope component $h_{1}$ :

$$
h_{1}=X(t)-m_{1}
$$

351 Using $h_{1}$ as the new time series, this process is repeated $k$ times until the standard deviation of $352 h_{1 k}, \sigma_{k}$, is less than 0.8 . This value was selected as it allows for a relatively small number of

353 IMF components, whilst still capturing signal complexity:

$$
\sigma_{k}=\sum_{t=0}^{T} \frac{\left|h_{k-1}(t)-h_{k}(t)\right|^{2}}{h_{k-1}^{2}(t)}
$$

Thus, $h_{1 k}$ is defined as the first IMF component of the data:

$$
c_{1}=h_{1 k}
$$

Typically, $c_{1}$ contains the highest frequency component of $X(t)$. Subsequent IMF, which contain longer period data, are then calculated from the residual $r_{n}$ where:

$$
r_{n}=r_{n-1}-c_{n}
$$

360 The sifting process is stopped when $r_{n}$ becomes a monotonic function from which no new IMF

361 can be extracted. Consequently, the test data is decomposed into $n$ empirical modes where

$$
X(t)=\sum_{j=1}^{n} c_{j}+r_{n}
$$



component is calculated as:

$$
H\left(c_{n}\right)(t)=\frac{1}{\pi} \int_{-\infty}^{\infty} \frac{c_{n}(\tau)}{t-\tau} d \tau
$$

Where $\tau$ is the width of the analysis window. The phase angle $\theta$,

$$
\theta=\operatorname{Im}\left(\log \left(H\left(c_{n}\right)\right)\right)
$$

is then used to compute the instantaneous frequency content $f_{\text {inst }}$ as

$$
f_{\text {inst }}=\frac{\Delta(\theta) / T s}{2 * \pi}
$$

where $T s$ is the sampling period. To characterise the data for use in classification, each step of the vector $\operatorname{freq}(t)$ is then defined as the $f_{\text {inst }}(t)$ of the highest $A_{\text {inst }}(t)$ of all IMF components in $X(t)$. Thus, freq is simply a high-resolution vector of the dominant frequency content through time.

\section{Permutation Entropy}

The Permutation Entropy method describes the uncertainty and the degree of irregularity in a random series. To compute the empirical PE (refer to Unakafova and Keller, (2013) for more details), ordinal patterns of the AE are obtained with delay of $\tau=1$, indicating a distance of 1 between points in patterns and an order of $d=5$, meaning patterns contain 6 points $(d+1)$. With a high degree of overlap between points, it is possible to use all of the information about order relations between points in the $\mathrm{AE}$ waveform. The distribution of ordinal patterns are obtained by the simple enumeration of the type of pattern. The PE, computed in a sliding time

382 window of 30 points to reflect the complexity in the time series, is calculated at time $t$ as:

$$
P E_{d}^{\mathcal{\tau}}(t)=-\sum_{j=0}^{(d+1) !-1} \frac{q_{j}}{M} \ln \frac{q_{j}}{M}=\ln M-\frac{1}{M} \sum_{j=0}^{(d+1) !-1} q_{j} \ln q_{j}
$$


where $q_{j}=\#\{k \in\{t, t-1, \ldots, t-M+1\}$ has the ordinal pattern $j\}$ (with $0 \ln 0:=0$ ) and $M$ is defined as the number of ordinal patterns in the window.

386

387

388

390

391

392

393

394

395

396

397

398

399

400

401

402

403

404

405

406

\section{References}

Akaike, H. (1973). Information theory as an extension of the maximum likelihood principle. Á In: Petrov, BN and Csaki, F. In Second International Symposium on Information Theory. Akademiai Kiado, Budapest, pp. 276Á281.

Baud, P., \& Meredith, P. (1997). Damage accumulation during triaxial creep of Darley Dale sandstone from pore volumometry and acoustic emission. International Journal of Rock Mechanics and Mining Sciences, 34(3-4), 24-e1.

Benson, P. M., Thompson, B. D., Meredith, P. G., Vinciguerra, S., \& Young, R. P. (2007). Imaging slow failure in triaxially deformed Etna basalt using 3D acoustic-emission location and X-ray computed tomography. Geophysical Research Letters, 34(3). https://doi.org/10.1029/2006g1028721

Benson, P. M., Vinciguerra, S., Meredith, P. G., \& Young, R. P. (2010). Spatio-temporal evolution of volcano seismicity: A laboratory study. Earth and Planetary Science Letters, 297(1-2), 315-323.

Benson, P. M., Austria, D. C., Gehne, S., Butcher, E., Harnett, C. E., Fazio, M., et al. (2019). Laboratory simulations of fluid-induced seismicity, hydraulic fracture, and fluid flow. Geomechanics for Energy and the Environment, 100169. https://doi.org/10.1016/j.gete.2019.100169

Bohse, J. (2000). Acoustic emission characteristics of micro-failure processes in polymer blends and composites. Composites Science and Technology, 60(8), 1213-1226. https://doi.org/10.1016/S0266-3538(00)00060-9 
Chen, Y., Zhang, G., Bai, M., Zu, S., Guan, Z., \& Zhang, M. (2019). Automatic Waveform

408

409

410

411

412

413

414

415

416

417

418

419

420

421

422

423

424

425

426

427

428

429

430 Classification and Arrival Picking Based on Convolutional Neural Network. Earth and Space Science, 6(7), 1244-1261. https://doi.org/10.1029/2018EA000466

Comanducci, L., Cobos, M., Antonacci, F., \& Sarti, A. (2020). Time Difference of Arrival Estimation from Frequency-Sliding Generalized Cross-Correlations Using Convolutional Neural Networks. ArXiv:2002.00641 [Cs, Eess]. Retrieved from http://arxiv.org/abs/2002.00641

Derakhshani, R., \& Schuckers, S. A. C. (2004). Continuous time delay neural networks for detection of temporal patterns in signals. In 2004 IEEE International Joint Conference on Neural Networks (IEEE Cat. No.04CH37541) (Vol. 4, pp. 2723-2728 vol.4). https://doi.org/10.1109/IJCNN.2004.1381082

Fazio, M. (2017, January). Dynamic Laboratory Simulations of Fluid-Rock Coupling with Application to Volcano Seismicity and Unrest (PhD Thesis). University of Portsmouth, School of Earth and Environmental Sciences.

Fazio, M., Alparone, S., Benson, P. M., Cannata, A., \& Vinciguerra, S. (2019). Genesis and mechanisms controlling tornillo seismo-volcanic events in volcanic areas. Scientific Reports, 9(1), 1-11.

G, S., K p, S., \& R, V. (2018). Automated detection of cardiac arrhythmia using deep learning techniques. Procedia Computer Science, 132, 1192-1201. https://doi.org/10.1016/j.procs.2018.05.034

Gehne, S. (2018). A laboratory study of fluid-driven tensile fracturing in anisotropic rocks. University of Portsmouth.

Grosse, C. U., \& Ohtsu, M. (2008). Acoustic emission testing. Springer Science \& Business Media. 
431 Guo, C., Zhu, T., Gao, Y., Wu, S., \& Sun, J. (2020). AEnet: Automatic Picking of P-Wave First Arrivals Using Deep Learning. IEEE Transactions on Geoscience and Remote Sensing, 1-11. https://doi.org/10.1109/TGRS.2020.3010541

434

435

436

437

438

439

440

441

442

443

444

445

446

447

Guoping, Z., Yanchun, W., \& Rongliang, S. (2004). An improved method for first arrival pickup using energy ratio. Geophysical Prospecting for Petroleum, 43(4), 345-347.

Haffner, P., \& Waibel, A. (1992). Multi-state time delay networks for continuous speech recognition. In Advances in neural information processing systems (pp. 135-142).

Heap, M. J., Baud, P., Meredith, P. G., Bell, A. F., \& Main, I. G. (2009). Time-dependent brittle creep in Darley Dale sandstone. Journal of Geophysical Research: Solid Earth, 114(B7).

Høgsberg, J., \& Krenk, S. (2015). Balanced calibration of resonant piezoelectric RL shunts with quasi-static background flexibility correction. Journal of Sound and Vibration, 341, 16-30. https://doi.org/10.1016/j.jsv.2014.12.006

Huang, N. E., Shen, Z., Long, S. R., Wu, M. C., Shih, H. H., Zheng, Q., et al. (1998). The empirical mode decomposition and the Hilbert spectrum for nonlinear and nonstationary time series analysis. Proceedings of the Royal Society of London. Series A: Mathematical, Physical and Engineering Sciences, 454(1971), 903-995.

Jiang, T., \& Zheng, J. (2020). Automatic Phase Picking From Microseismic Recordings Using Feature Extraction and Neural Network. IEEE Access, 8, 58271-58278. https://doi.org/10.1109/ACCESS.2020.2982184

Lockner, D. A., Byerlee, J. D., Kuksenko, V., Ponomarev, A., \& Sidorin, A. (1992). Observations of quasistatic fault growth from acoustic emissions. In International Geophysics (Vol. 51, pp. 3-31). Elsevier.

454 Nakamura, Y., Veach, C. L., \& McCauley, B. O. (1972). Amplitude distribution of acoustic 
emission signals. In Acoustic Emission. ASTM International.

456

457

458

459

460

461

462

463

464

465

466

467

468

469

470

471

472

473

474

475

476

477

478

Peddinti, V., Chen, G., Manohar, V., Ko, T., Povey, D., \& Khudanpur, S. (2015). Jhu aspire system: Robust lvesr with tdnns, ivector adaptation and rnn-lms. In 2015 IEEE Workshop on Automatic Speech Recognition and Understanding (ASRU) (pp. 539546). IEEE.

Pomponi, E., \& Vinogradov, A. (2013). A real-time approach to acoustic emission clustering. Mechanical Systems and Signal Processing, 40(2), 791-804.

Ramírez-Rojas, A., Flores-Márquez, E., Sarlis, N., \& Varotsos, P. (2018). The complexity measures associated with the fluctuations of the entropy in natural time before the deadly México M8. 2 earthquake on 7 September 2017. Entropy, 20(6), 477.

Read, M. D., Ayling, M. R., Meredith, P. G., \& Murrell, S. A. F. (1995). Microcracking during triaxial deformation of porous rocks monitored by changes in rock physical properties, II. Pore volumometry and acoustic emission measurements on water-saturated rocks. Tectonophysics, 245(3), 223-235. https://doi.org/10.1016/0040-1951(94)00236-3

Ródenas, J., García, M., Alcaraz, R., \& Rieta, J. (2015). Wavelet entropy automatically detects episodes of atrial fibrillation from single-lead electrocardiograms. Entropy, 17(9), $6179-6199$.

Scholey, J. J., Wilcox, P. D., Wisnom, M. R., \& Friswell, M. I. (2010). Quantitative experimental measurements of matrix cracking and delamination using acoustic emission. Composites Part A: Applied Science and Manufacturing, 41(5), 612-623. https://doi.org/10.1016/j.compositesa.2010.01.008

Sharma, R., Pachori, R., \& Acharya, U. (2015). Application of entropy measures on intrinsic mode functions for the automated identification of focal electroencephalogram signals. Entropy, 17(2), 669-691. 
479 Snyder, D., Garcia-Romero, D., \& Povey, D. (2015). Time delay deep neural network-based universal background models for speaker recognition. In 2015 IEEE Workshop on Automatic Speech Recognition and Understanding (ASRU) (pp. 92-97). IEEE.

482

483

484

485

486

487

488

489

490

491

492

493

494

495

496

497

498

499

500

Stanchits, S., Vinciguerra, S., \& Dresen, G. (2006). Ultrasonic Velocities, Acoustic Emission Characteristics and Crack Damage of Basalt and Granite. Pure and Applied Geophysics, 163(5), 975-994. https://doi.org/10.1007/s00024-006-0059-5

Tobias, A. (1976). Acoustic-emission source location in two dimensions by an array of three sensors. Non-Destructive Testing, 9(1), 9-12. https://doi.org/10.1016/00291021(76)90027-X

Unakafova, V., \& Keller, K. (2013). Efficiently measuring complexity on the basis of realworld data. Entropy, 15(10), 4392-4415.

Waibel, A., Hanazawa, T., Hinton, G., Shikano, K., \& Lang, K. J. (1995). Phoneme recognition using time-delay neural networks. Backpropagation: Theory, Architectures and Applications, 35-61.

Yao, Z., Wang, Z., Liu, W., Liu, Y., \& Pan, J. (2020). Speech emotion recognition using fusion of three multi-task learning-based classifiers: HSF-DNN, MS-CNN and LLD-RNN. Speech Communication, 120, 11-19. https://doi.org/10.1016/j.specom.2020.03.005

Zhang, H. L., Zhu, G. M., \& Wang, Y. H. (2013). Automatic microseismic event detection and picking method. Geophysical and Geochemical Exploration, 37(2), 269-273.

Zhu, W., \& Wong, T. (1997). The transition from brittle faulting to cataclastic flow: Permeability evolution. Journal of Geophysical Research: Solid Earth, 102(B2), 30273041.

Zoukaneri, I., \& Porsani, M. J. (2015). A combined Wigner-Ville and maximum entropy method for high-resolution time-frequency analysis of seismic data. Geophysics, 80(6), 


\section{Author Mailing Addresses}

Valperga Caluso, 35, 10125 Turin, Italy; thomas.king@edu.unito.it.

511 Germany; ldesiena@uni-mainz.de.

513 Valperga Caluso, 35, 10125 Turin, Italy; sergiocarmelo.vinciguerra@unito.it.

\section{Figure Legend}

515 Figure 1: Input parameters used in model training. Data classifications, indicated above, are noise and signal. a)

516 Seismic envelope. High values typically denote signal, however, low amplitude data is poorly characterised. b)

517 Dominant frequency content of the AE waveform. Red and blue indicates high and low frequency content

518 respectively. Pre-signal noise is characterised by high frequency and the signal itself with low values. c)

519 Permutation energy behaves similarly to seismic envelope but the trend is opposite with low values identifying signal. Even at low amplitudes, permutation entropy detects coherancy in frequency content.

521 Figure 2: Flowchart detailing key elements of the training loop.

Figure 3: AE signal (black), neural network model output (NN, red) and amplitude threshold (AT, blue) highlight the discrepencies in picking quality between the two methods at low amplitudes. Strongly scattered or emergent waveforms are frequently misspicked due to late arrivals in the coda. The AT method also misses many low amplitude arrivals that the $\mathrm{NN}$ is still able to detect. 
530 Figure A2: Velocity data, stress-strain curve and raw AE count for Darley Dale Sandstone deformed at $20 \mathrm{MPa}$ 531 confning pressure.

532

533

\section{Figures (In order of appearance)}
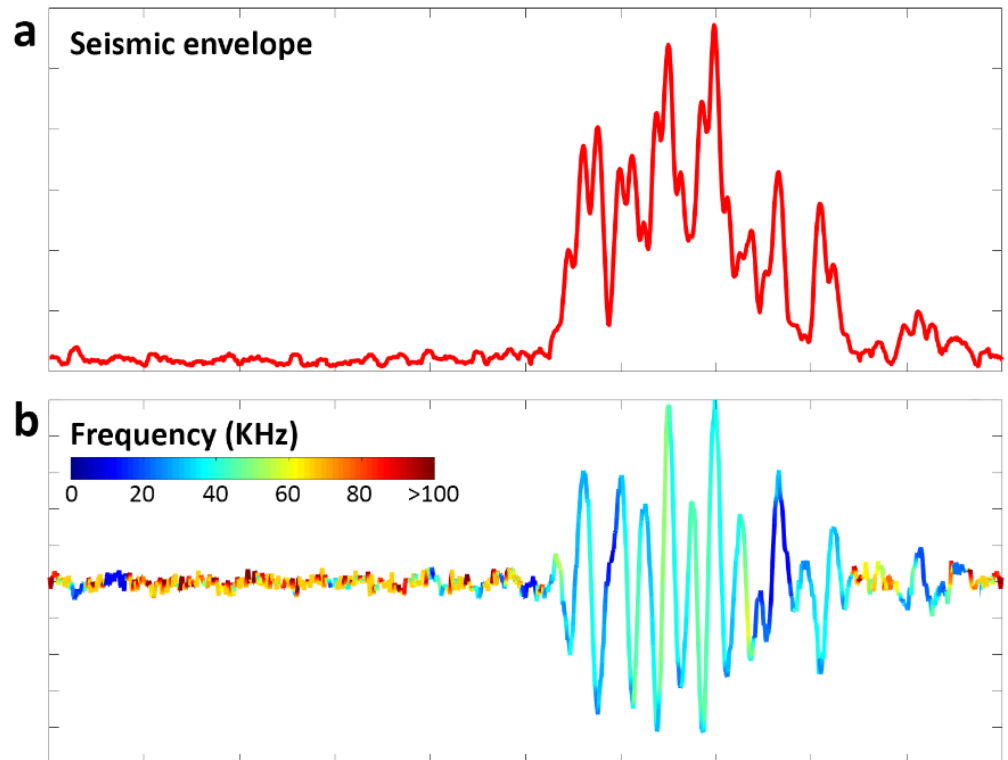

\section{Permutation entropy}

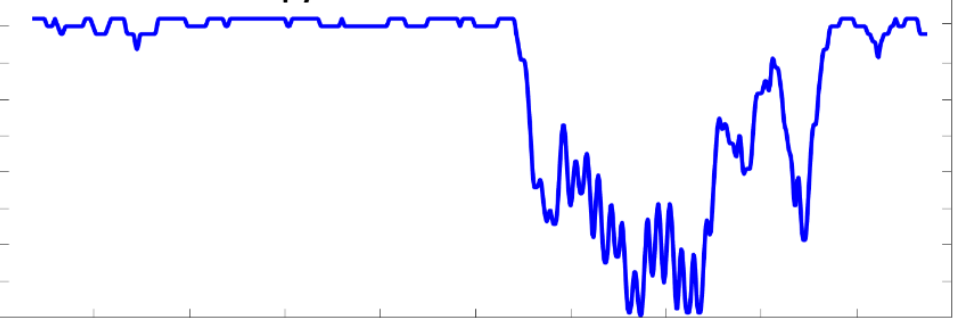

536 Figure 1: Input parameters used in model training. Data classifications, indicated above, are noise and signal. a)

537 Seismic envelope. High values typically denote signal, however, low amplitude data is poorly characterised. b)

538 Dominant frequency content of the AE waveform. Red and blue indicates high and low frequency content

539 respectively. Pre-signal noise is characterised by high frequency and the signal itself with low values. c)

540 Permutation energy behaves similarly to seismic envelope but the trend is opposite with low values identifying

541 signal. Even at low amplitudes, permutation entropy detects coherancy in frequency content. 


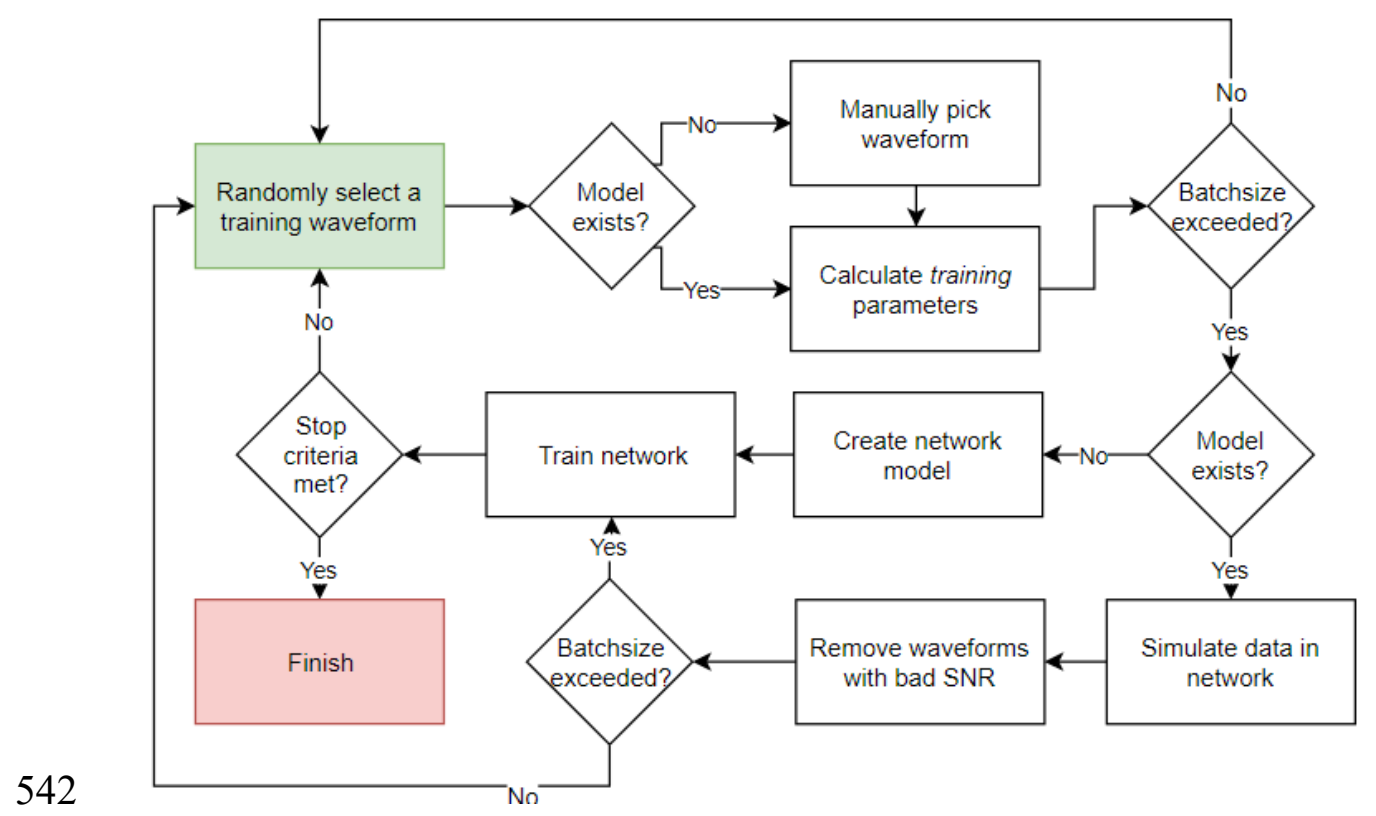

543 Figure 2: Flowchart detailing key elements of the training loop. 

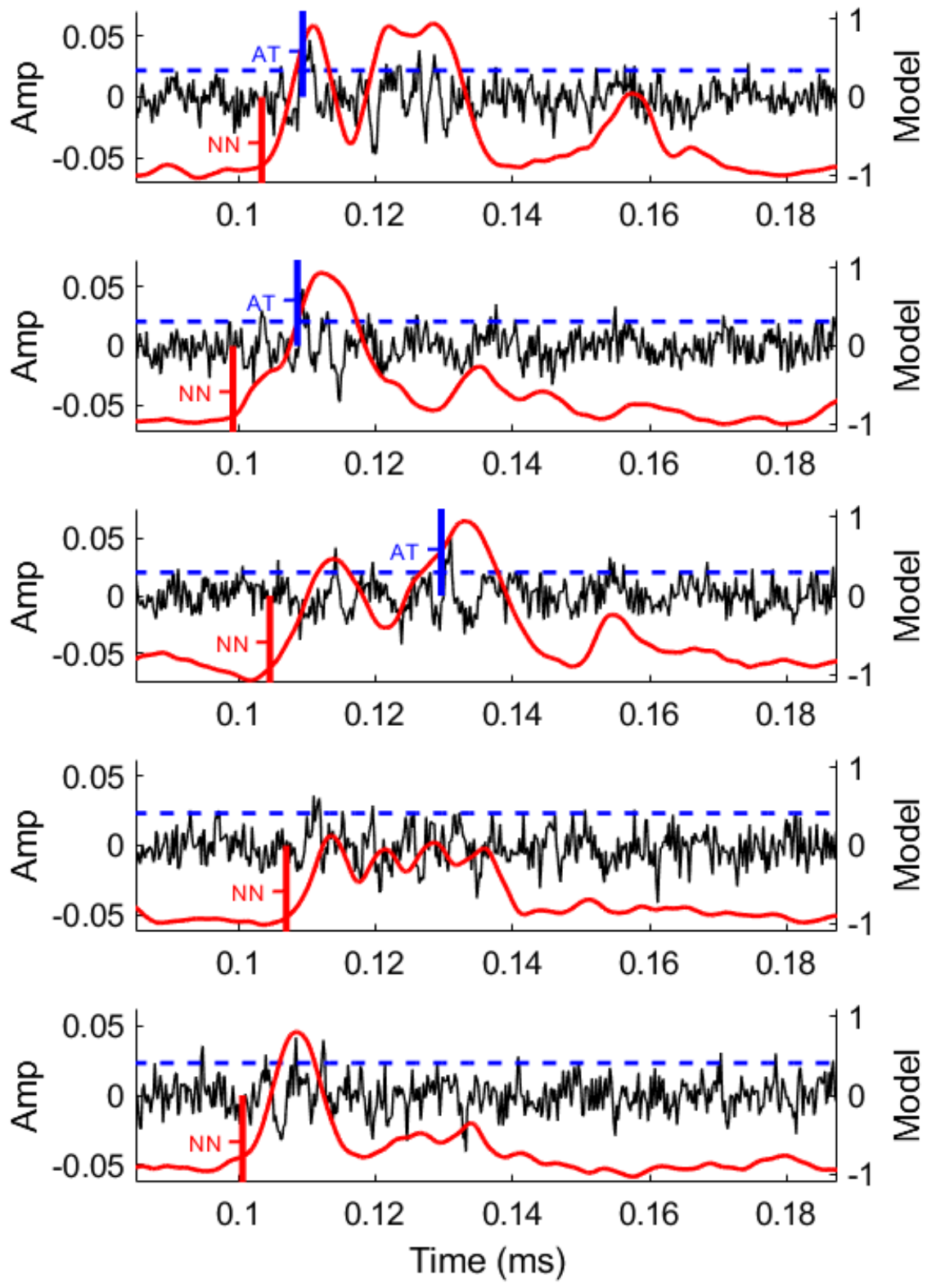

545 Figure 3: AE signal (black), neural network model output (NN, red) and amplitude threshold (AT, blue) highlight 546 the discrepencies in picking quality between the two methods at low amplitudes. Strongly scattered or emergent

547 waveforms are frequently misspicked due to late arrivals in the coda. The AT method also misses many low 548 amplitude arrivals that the NN is still able to detect. 

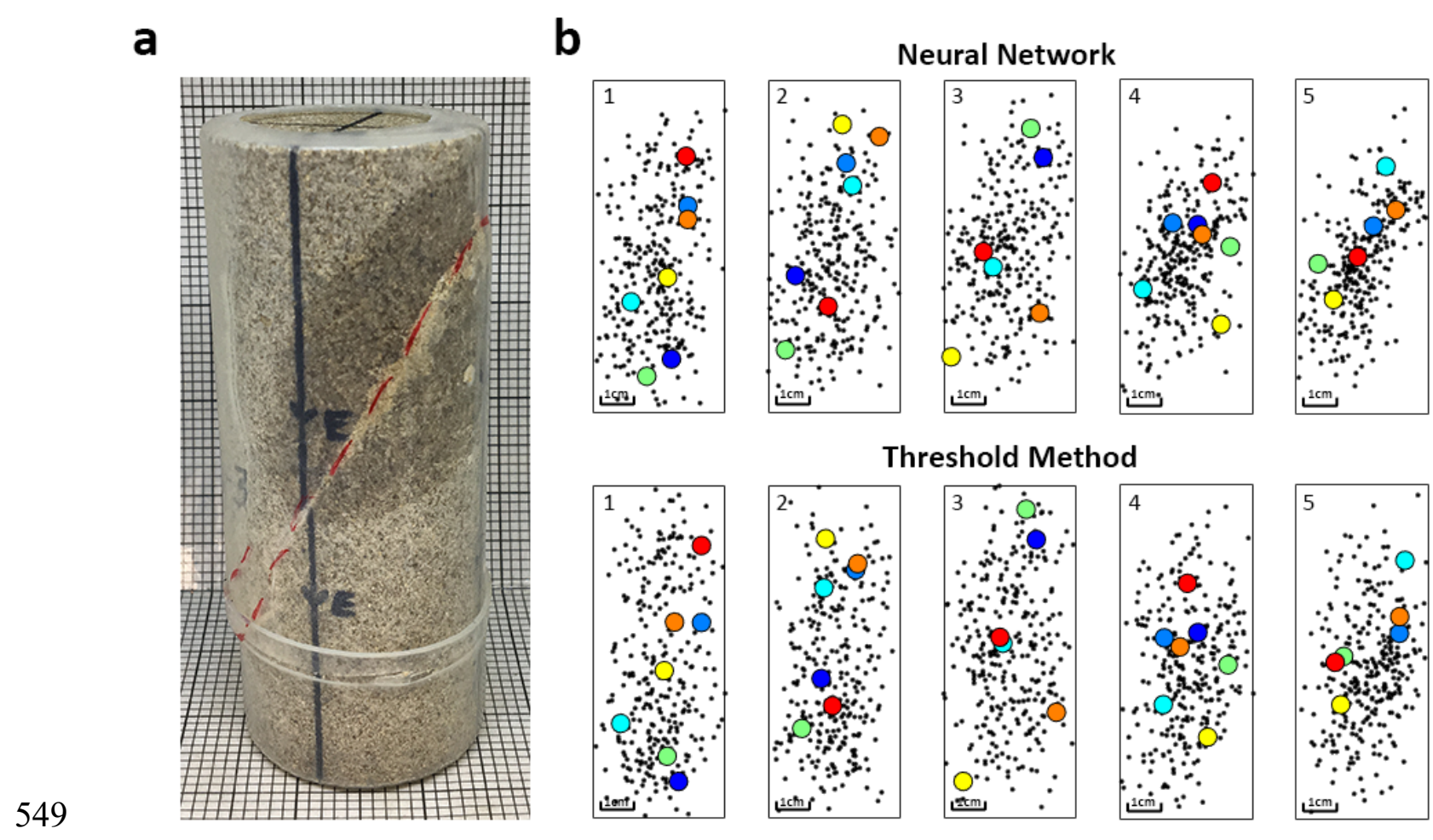

550 Figure 4: a) Post-failure imaging of the sample highlights a singular failure plane propagating to the lower left. b)

551 AE sources that are located by both methodologies (black dots). Example locations (corresponding coloured dots)

552 highlight the discreprency in source location due to the different picking methodologies.

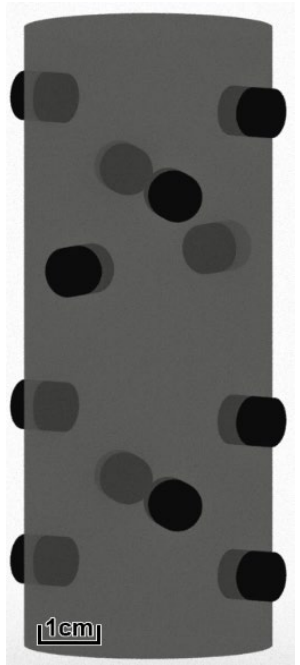




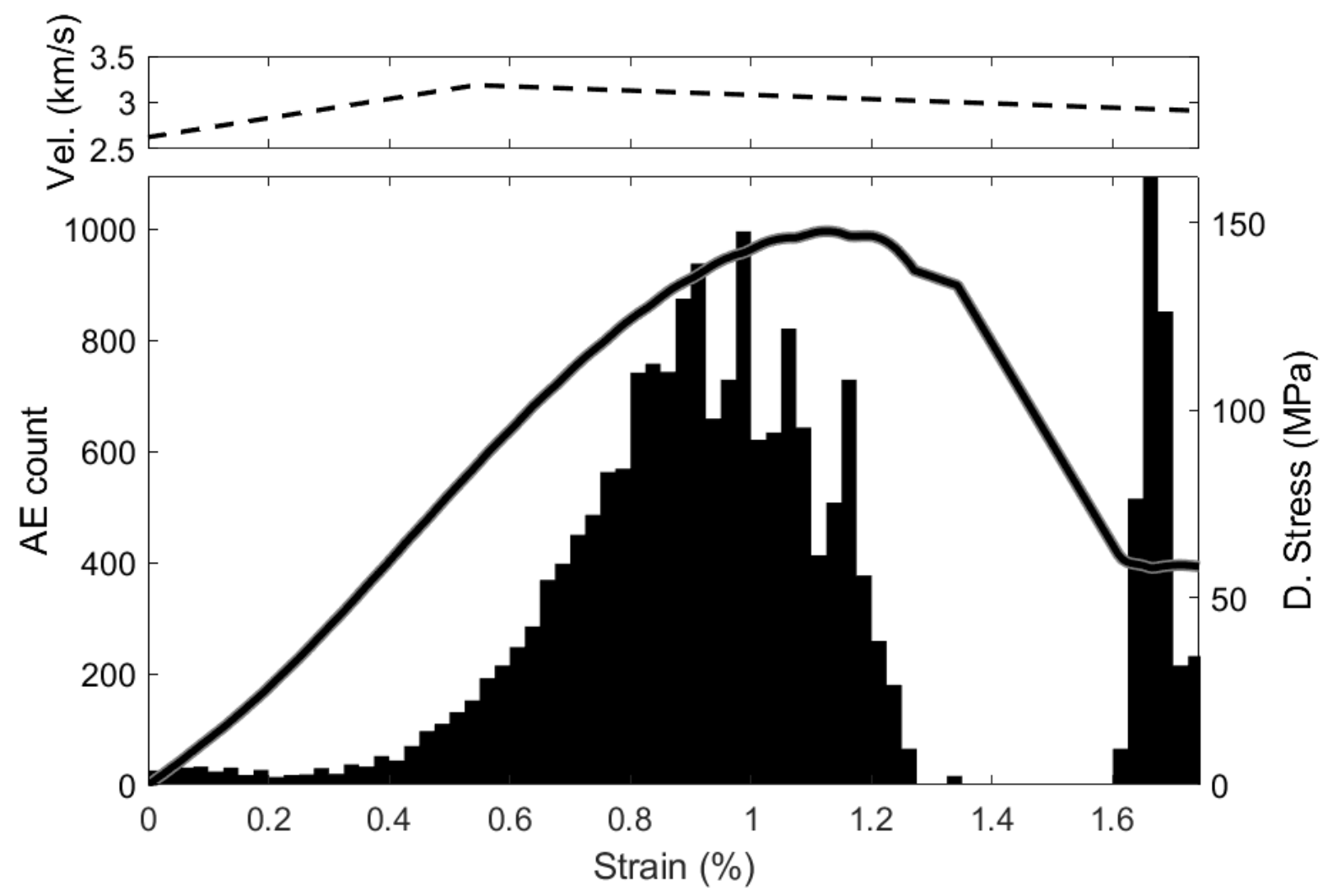

558 Figure A2: Velocity data, stress-strain curve and raw AE count for Darley Dale Sandstone deformed at 20 MPa 559 confning pressure. Brittle failure ocucrs at approximately $1,2 \%$ strain. 